\title{
Neutron diffraction evidence for kinetic arrest of first-order magneto-structural phase transitions in some functional magnetic materials
}

\author{
V Siruguri ${ }^{1}$, P D Babu ${ }^{1}$, S D Kaushik ${ }^{1}$, Aniruddha Biswas ${ }^{2}$, S \\ K Sarkar ${ }^{2}$, Madangopal Krishnan ${ }^{2}$ and $\mathbf{P}$ Chaddah $^{3}$ \\ ${ }^{1}$ UGC-DAE Consortium for Scientific Research Mumbai Centre, Bhabha Atomic \\ Research Centre, Mumbai-400085, India \\ ${ }^{2}$ Glass and Advanced Materials Division, Bhabha Atomic Research Centre, \\ Mumbai-400085, India \\ ${ }^{3}$ UGC-DAE Consortium for Scientific Research, University Campus, Khandwa Road, \\ Indore-452001, India \\ E-mail: siruguri@csr.res.in
}

\begin{abstract}
.
Neutron diffraction measurements, performed in presence of an external magnetic field, have been used to show structural evidence for the kinetic arrest of the firstorder phase transition from (i) the high temperature austenite phase to the low temperature martensite phase in the magnetic shape memory alloy $\mathrm{Ni}_{37} \mathrm{Co}_{11} \mathrm{Mn}_{42.5}$ $\mathrm{Sn}_{9.5}$, (ii) the higher temperature ferromagnetic phase to the lower temperature antiferromagnetic phase in the half-doped charge ordered compound $\mathrm{La}_{0.5} \mathrm{Ca}_{0.5} \mathrm{MnO}_{3}$ and (iii) the formation of glass-like arrested states in both compounds. The cooling and heating under unequal fields protocol has been used to establish phase coexistence of metastable and equilibrium states, and also to demonstrate the devitrification of the arrested metastable states in the neutron diffraction patterns. We also explore the field-temperature dependent kinetic arrest line $\mathrm{T}_{K}(\mathrm{H})$, through the transformation of the arrested phase to the equilibrium phase. This transformation has been observed isothermally in reducing $\mathrm{H}$, as also on warming in constant $\mathrm{H}$. $\mathrm{T}_{K}$ is seen to increase as $\mathrm{H}$ increases in both cases, consistent with the low- $\mathrm{T}$ equilibrium phase having lower magnetization.
\end{abstract}

PACS numbers: 61.05.fm, 75.25.-j, 75.60.Nt

Submitted to: J. Phys.: Condens. Matter 


\section{Introduction}

A number of functional magnetic materials like CMR manganites, magnetic shape memory alloys, intermetallics, multiferroics, etc., exhibit first order phase transitions (FOPT) as a function of temperature, pressure or magnetic field. While the microscopic nature of these FOPT has been extensively studied using temperature and pressure, the use of magnetic field as a useful thermodynamic variable in exploring these FOPT at an atomic scale is not very common. However, in recent times, the study of first order transformation kinetics of these functional magnetic materials under the influence of a magnetic field has been subject of intense experimental research [1, 2, 3, 4, 5]. In these materials, typically, the high temperature magnetic phase can be kinetically arrested when cooled under an appropriate external magnetic field, thereby inhibiting the transformation to the low temperature equilibrium phase. This kinetically arrested state has been shown to have coexisting phases of magnetically ordered metastable and equilibrium states and such a material has been termed [6, 7] as a 'magnetic glass'. These studies attempt to investigate those regimes in knowledge space which have been hitherto unexplored, by using magnetic field as a useful thermodynamic variable. While the experimental techniques used in these studies are mostly based on transport and magnetization measurements, atomic scale measurements delineating the crystalline and magnetic structures as a function of magnetic field, for which neutron diffraction is a unique probe, have not been carried out so far. Certain classes of ferromagnetic shape memory alloys (FSMA) like Co-doped NiMnSn, NiMnIn and NiMnAl undergo a kinetic arrest of the first-order austenite to martensite transition [6, 8, 9, 10]. In these materials, the higher temperature austenite phase has a higher ferromagnetic moment as compared to the lower temperature martensite phase and this manifests itself as a sharp drop in magnetization in a $\mathrm{M}$ versus $\mathrm{T}$ measurement. By increasing the magnetic field of measurement, it has been observed that the kinetics associated with this first order transformation get hindered and beyond a critical field, they get completely arrested. Hence, there will be regions of field-temperature space where there will be a phase coexistence of metastable (arrested) or glass-like arrested states (GLAS) and equilibrium (transformed) states. If the lower temperature equilibrium (transformed) state has a lower magnetization value than the high temperature austenite phase, it has been noted earlier that by cooling in a certain higher field $\left(\mathrm{H}_{c}\right)$ and warming in a lower field $\left(\mathrm{H}_{w}\right)$ would lead to a de-arrest or devitrification of the GLAS [11]. On further warming, the devitrified state would undergo a reverse magnetic and structural transition to the high temperature, high moment austenite phase. This novel protocol of cooling and heating in unequal fields (CHUF) [11] offers an unambiguous method to observe if the glass-like arrested state devitrifies, thereby qualifying it to be called a magnetic glass. While the transitions in this material are predominantly structural, kinetic arrest has also been observed in materials which undergo a ferromagnetic to antiferromagnetic transition, like, for example, the archetypal half-doped, charge-ordered manganite $\mathrm{La}_{0.5} \mathrm{Ca}_{0.5} \mathrm{MnO}_{3}$ (LCMO) [12. This compound is paramagnetic at room temperature, and upon cooling, 
it first enters a ferromagnetic $(\mathrm{FM})$ state at $\sim 225 \mathrm{~K}$ and then undergoes a FOPT into an antiferromagnetic $(\mathrm{AFM})$ state at $\sim 150 \mathrm{~K}[13$. Transport and magnetization measurements employing similar measurement protocols show that the ferromagnetic phase can be kinetically arrested when cooled under an appropriate magnetic field through the FM-AFM transition, resulting in coexisting phases of metastable (arrested) FM states and equilibrium (transformed) AFM states [14]. The percentages of these phases would depend on the magnitude of the cooling field. CHUF protocol has been employed in the case of this compound also to demonstrate, through magnetization measurements, the devitrification of these glass-like arrested metastable states [12]. While transport and magnetization measurements do not provide information about the structures at the microscopic scale, neutron diffraction is a powerful tool which can not only give information about the crystalline structure but also the magnetic structure. In particular, antiferromagnetic ordering of spins gives rise to unique reflections in neutron diffraction whose origin is purely magnetic in nature. Therefore, it is expected that neutron diffraction in presence of an external magnetic field, when carried out using the above measurement protocol, would give unambiguous information about coexisting phases, crystalline and magnetic, in a material.

We present here neutron diffraction (ND) studies, in presence of an external magnetic field over a wide region of the field-temperature $(\mathrm{H}, \mathrm{T})$ space, on two widely different materials (i) the ferromagnetic shape memory (FSMA) alloy $\mathrm{Ni}_{37} \mathrm{Co}_{11} \mathrm{Mn}_{42.5} \mathrm{Sn}_{9.5}$ and (ii) half-doped manganite $\mathrm{La}_{0.5} \mathrm{Ca}_{0.5} \mathrm{MnO}_{3}$. The former, on cooling, undergoes a FOPT from a high moment, austenite phase to a low moment, martensite phase while the latter compound undergoes a predominantly first order magnetic transition from a ferromagnetic state to an antiferromagnetic state on cooling. We show evidence, for the first time, for the kinetic arrest of the first order structural and magnetic phase transitions in these compounds. In $\mathrm{Ni}_{37} \mathrm{Co}_{11} \mathrm{Mn}_{42.5} \mathrm{Sn}_{9.5}$, the first order austenite to martensite transition gets kinetically arrested when cooled under an appropriate magnetic field, and on heating from $2 \mathrm{~K}$ to room temperature in a different field, a subsequent devitrification of the arrested metastable austenite state to the martensite state takes place, followed by the reentrant martensite-to-austenite transition. Similarly, in the half doped $\mathrm{La}_{0.5} \mathrm{Ca}_{0.5} \mathrm{MnO}_{3}$, we show that the higher temperature ferromagnetic phase gets kinetically arrested on cooling under a magnetic field and when subsequently heated under a different field, the metastable arrested ferromagnetic state devitrifies into the stable antiferromagnetic state before reentering the higher temperature ferromagnetic state. The phase coexistence of the arrested metastable and equilibrium states by employing the CHUF protocol is demonstrated. Finally, we explain the $(\mathrm{H}, \mathrm{T})$ phase diagram for the two compounds using the $\mathrm{T}_{K}(\mathrm{H})$ line and attempt to draw analogies with the pressure-temperature $(\mathrm{P}, \mathrm{T})$ phase diagrams for metallic liquid germanium [15] and glassy water [16]. This study also brings out the versatility of magnetic field over hydrostatic pressure in that it is much easier to vary magnetic field as compared to pressure. 


\section{Experimental details}

The $\mathrm{Ni}_{37} \mathrm{Co}_{11} \mathrm{Mn}_{42.5} \mathrm{Sn}_{9.5}$ buttons were made by vacuum arc melting high purity (99.99\%) elements in appropriate proportion. The buttons were solutionized at $1273 \mathrm{~K}$ for $24 \mathrm{~h}$ in sealed quartz ampoule. High temperature Differential Scanning Calorimetry (DSC) was used to confirm the two-phase melting behavior of this alloy and an extended homogenization treatment was given to ensure structural and chemical homogeneity. Characterization of this alloy has been carried out using Optical and Scanning Electron Microscopy, Electron Probe Microanalysis, XRD and neutron diffraction (ND), Transmission Electron Microscopy (TEM), DSC and DC magnetization. Bulk cylindrical specimens of $5 \mathrm{~mm}$ diameter were prepared for ND using electro-discharge machining, as powder samples do not undergo martensitic transformation in this alloy [5]. Nevertheless, one powder specimen was also prepared by crushing a part of the treated button and checked for room temperature structure. Specimens for TEM were prepared by slicing discs from an electro-discharge machined cylindrical rod of $3 \mathrm{~mm}$ diameter, followed by grinding and jet polishing with a Struers Tenupol-5 at $233 \mathrm{~K}$, using a 10 vol \% perchloric acid in methanol electrolyte. For ND measurements, the bulk cylindrical specimen was directly attached to the sample stick and inserted into the cryomagnet. The $\mathrm{La}_{0.5} \mathrm{Ca}_{0.5} \mathrm{MnO}_{3}$ sample was prepared by the conventional solid-state route and the single phase nature was confirmed using XRD whose pattern matched well with literature reports. Polycrystalline powder sample was compacted into pellets and inserted into the vanadium sample holders. This avoided any preferential orientations of the grains under a magnetic field. The ND patterns were collected using the positionsensitive detector based focusing crystal diffractometer installed by the UGC-DAE CSR Mumbai Centre [17] at the Dhruva reactor, Trombay, at a wavelength of $1.48 \AA$ in the temperature range of $2 \mathrm{~K}$ to $300 \mathrm{~K}$ under different conditions of applied magnetic field up to 7 Tesla. It was observed that the cylindrical FSMA sample consisted of extremely large grains which manifest themselves in almost single crystal-like behavior in the diffraction patterns. Hence, the sample was oriented such that two major reflections of the austenite phase at room temperature, namely, (111) and (200) were clearly visible

in the diffraction patterns. The sample was locked in this orientation for all subsequent measurements. DC magnetization was measured using a commercial 9 T PPMS-VSM (make Quantum Design).

\section{Results and discussion}

\subsection{Ferromagnetic shape memory alloy $\mathrm{Ni}_{37} \mathrm{Co}_{11} \mathrm{Mn}_{42.5} \mathrm{Sn}_{9.5}$}

3.1.1. Alloy characterization Figure 1 displays the high temperature DSC plots of this alloy. It clearly shows the two-phase incongruent melting behavior. Earlier, Watchel et al [18] noted the presence of a peritectic reaction in the ternary $\mathrm{Ni}_{0.5} \mathrm{Mn}_{0.5-x} \mathrm{Sn}_{x}$ $(0 \leq x \leq 0.5)$ pseudo-binary phase diagram. More recently, Yuhasz et al [19] also reported the problem of chemical and structural inhomogeneity that was prevalent in 
the ternary Ni-Mn-Sn alloys and was caused by this incongruent melting. It was thus, very important to solutionize these alloys for an extended period of time to ensure single-phase and uniform microstructure. Microstructure in as-solutionized condition shows the parent L 2 phase (figure 2(a)), as confirmed by the $<110>$ SAD pattern and its simulated key (figure 2(b-c)).

3.1.2. DC Magnetization Figure 3 shows the field cooled cooling (FCC) and field cooled warming (FCW) magnetization data at several different fields taken on bulk sample of $\mathrm{Ni}_{37} \mathrm{Co}_{11} \mathrm{Mn}_{42.5} \mathrm{Sn}_{9.5}$. The observed thermal hysteresis in the FCC and FCW curves clearly indicates that there is a first order phase transition (FOPT) of high magnetization austenite phase to low magnetization martensite phase in the cooling cycle and the reverse transformation in the warming cycle. The magnitude of thermal hysteresis gradually increases as the field is raised and has maximum hysteresis for $\mathrm{H}=0.5 \mathrm{~T}$, and by $2 \mathrm{~T}$ field, the hysteresis disappears as the austenite to martensite transformation is completely hindered by the field and the austenite phase gets kinetically arrested at low temperatures. Such behavior was seen earlier [20] in similar type of compounds. The austenite to martensite transformation start temperature $\left(\mathrm{M}_{S} \sim 181 \mathrm{~K}\right)$ in the cooling cycle and the reverse martensite to austenite transformation finish temperature $\left(\mathrm{A}_{F} \sim 230 \mathrm{~K}\right)$ in the heating cycle, are nearly same for fields up to $0.5 \mathrm{~T}$. For $1.5 \mathrm{~T}$, both these temperatures are shifted towards low temperatures to about $145 \mathrm{~K}$ and $214 \mathrm{~K}$, respectively. In other words, the temperature regime over which the hysteresis persists is nearly the same for fields up $0.5 \mathrm{~T}$, and for $1.5 \mathrm{~T}$, it increases significantly although magnitude in terms of moment has come down. Both FCC and FCW curves merge and level off at low temperatures. The magnetization value at $5 \mathrm{~K}$ increases systematically with field as it will follow the $\mathrm{M}-\mathrm{H}$ curve of martensite phase and also the phase fraction of austenite that remains arrested increases with field.

Figure 4 shows the $\mathrm{M}$ versus $\mathrm{H}$ isotherms taken at different temperatures. The final temperatures, at which $\mathrm{M}-\mathrm{H}$ isotherms were recorded, are reached by cooling the sample directly from $350 \mathrm{~K}$ in each case. For $\mathrm{T}>200 \mathrm{~K}$, the sample is in austenite phase and shows a ferromagnetic MH with high magnetization value $(\sim 130-145 \mathrm{emu} / \mathrm{g})$. At $160 \mathrm{~K}$, the sample is in martensite phase and one observes a ferromagnetic $\mathrm{MH}$ isotherm with low magnetization value $(\sim 55 \mathrm{emu} / \mathrm{g})$. However, with increasing field, one observes a broad field-induced martensite to austenite transformation over a field range of $3 \mathrm{~T}-4 \mathrm{~T}$. This trend continues as temperature is lowered to $5 \mathrm{~K}$ and only the fields at which this transformation takes place go on increasing. At $5 \mathrm{~K}$, even a field of $9 \mathrm{~T}$ is not enough to transform the system completely into the austenite phase.

In figure 5, the field is raised to $9 \mathrm{~T}$ at $350 \mathrm{~K}$ and the sample is cooled to 5 $\mathrm{K}$ in field. Then, the field is isothermally reduced to zero at $5 \mathrm{~K}$ (red curve). This measurement shows that some devitrification of the arrested austenite phase takes place around 5 Tesla (inset of figure 5) as indicated by the drop in the magnetization which is still higher than the zero-field cooled (ZFC) magnetization at $5 \mathrm{~K}$. Next, the CHUF protocol was employed to examine the kinetic arrest of the metastable 
austenite phase and its devitrification to the equilibrium martensite phase and their phase coexistence (figure 6). The sample was cooled in different fields ranging from 0.1 $\mathrm{T}$ to $8 \mathrm{~T}$ down to $5 \mathrm{~K}$ and then the field is raised or lowered at $5 \mathrm{~K}$ to the value of the measuring field of $0.5 \mathrm{~T}$ and the measurements were carried out in the warming cycle. ZFC magnetization curve, in which the sample was first zer-field cooled down to $5 \mathrm{~K}$ and then the data were collected during the warming cycle in a field of $0.5 \mathrm{~T}$, is also shown for comparison. For cooling fields which are less than $0.5 \mathrm{~T}$, only one transition from a low magnetization phase to the high magnetization austenite phase is observed which indicates that the low magnetization phase is the equilibrium martensite phase. Whereas, for cooling fields that are greater than $0.5 \mathrm{~T}$, a sharp drop in the magnetization at low temperatures indicates that a devitrification is taking place from a glass-like arrested phase to an equilibrium martensite phase. On further increasing the temperature, a reverse transformation to the high temperature high magnetization austenite phase takes place. These observations are discussed along with the neutron diffraction measurements performed using the CHUF protocol in the next section.

3.1.3. Neutron Diffraction Neutron diffraction patterns of bulk cylindrical specimens show that the structure could be indexed to an $\mathrm{L} 2{ }_{1}$-structure with cell parameter of 5.957 A (figure 7). The bulk sample exhibited strong large grain characteristics behaving almost like a single-crystal. The bulk sample was therefore oriented in such a way that the (111) and (200) reflections of the $\mathrm{L} 2_{1}$ austenite phase were strongly visible in the ND pattern. The sample was cooled down to $2 \mathrm{~K}$ in this orientation and the peaks associated with the martensite phase appear. Though it is difficult to index the structure with the limited number of reflections, an attempt was made using a standard indexing program and it was observed that the martensite phase is $10 \mathrm{M}$ modulated with cell parameters $a=4.338 \AA, b=5.534 \AA$ and $c=21.21 \AA$ and $\beta=92.55^{\circ}$. Four reflections belonging to the martensite phase have been clearly identified and marked in Fig. 7. A weak martensite peak is also observed at $\sim 31$ degrees which could be indexed as the (020) reflection of the $10 \mathrm{M}$ martensite. On warming the sample to 300 $\mathrm{K}$, there is a reverse transformation to the austenite phase in the neighborhood of 230 $\mathrm{K}$. Next, a field of $7 \mathrm{~T}$ was employed at $300 \mathrm{~K}$ and the sample was cooled again to $2 \mathrm{~K}$ and it was observed that the transition to the martensite phase was completely hindered, resulting in a kinetically arrested metastable austenite phase as evident from the complete absence of any martensite reflections in the ND pattern at the bottom of figure 8. Employing the CHUF protocol, the sample was cooled in a field of $7 \mathrm{~T}$ from $300 \mathrm{~K}$ several times but warmed in different fields each time. The warming fields used were $0.5 \mathrm{~T}, 1.0 \mathrm{~T}, 1.5 \mathrm{~T}$ and $2 \mathrm{~T}$. When the field was reduced from $7 \mathrm{~T}$ to $0.5 \mathrm{~T}$ for the first warming cycle, it was observed that the arrested austenite phase starts to devitrify at $2 \mathrm{~K}$ in a field of $0.5 \mathrm{~T}$ itself (figure 8) and the expected martensite peaks start appearing. In the next CHUF cycle, when the field was reduced from $7 \mathrm{~T}$ to $1 \mathrm{~T}$ at $2 \mathrm{~K}$, the sample remains in a kinetically arrested state at $2 \mathrm{~K}$ (figure 9), as evident from the absence of any peaks attributable to martensite phase. Devitrification 
sets in at around $10 \mathrm{~K}$ as evidenced by the appearance of the $10 \mathrm{M}$ martensite lines mentioned above and progresses with increasing temperature up to $230 \mathrm{~K}$, beyond which the system re-enters the austenite phase. With warming fields of $1.0 \mathrm{~T}$ and above (figures 9-11), it is seen that the temperature at which the devitrification sets in, increases with increase in the warming field. It is also observed that at this higher warming field, the intensity of the $10 \mathrm{M}$ martensite lines decreases implying that the phase fraction of devitrified phase is much lower. However, it is clear from figure 3, that cooling and warming in fields greater than $2 \mathrm{~T}$ would cause the transition to be completely arrested and no devitrification would be observed at any temperature below the standard martensite to austenite transition temperature. However, it must be kept in mind that while magnetization data might indicate a complete arrest of the phase, the same data may not be able to detect the presence of a small amount of the converted martensite phase and such a phase could be picked up by neutron diffraction. This evident from the very low intensity lines of the martensite phase in figure 11. It could be reasonably expected that there would be a complete arrest of the autenite phase at a higher field. It is important to note here that the quantity $\left(\mathrm{H}_{c}-\mathrm{H}_{w}\right)$ which is difference between the cooling field $\left(\mathrm{H}_{c}\right)$ and the warming field $\left(\mathrm{H}_{w}\right)$ is always positive and in the present case, it becomes a precondition to (i) observe the de-arrest of the metastable austenite phase which is similar to the phenomenon of devitrification of a conventional glass upon heating and (ii) the transformation of the devitrified equilibrium state to the high temperature austenite phase which is analogous to melting of devitrified glass. In figure 12, the temperature $\mathrm{T}_{K}$ at which devitrification, marked by the appearance of Bragg reflections attributable to the equilibrium martensite phase, sets in, is plotted against the value of the warming field $\mathrm{H}_{w}$. It is observed that $\mathrm{T}_{K}$ increases with $\mathrm{H}_{w}$. The set of temperature points $\mathrm{T}_{K}$ collectively form the kinetic arrest line which, when traversed across in the heating cycle, would result in the de-arrest of the metastable austenite phase to the equilibrium martensite phase. The line also indicates that at lower warming fields, one would have a higher phase fraction of the martensite phase when compared to higher warming fields. In order to substantiate this, the integrated intensity of $(103)_{M}$ reflection at $140 \mathrm{~K}$, which is the temperature at which the martensite phase fraction is expected to be at its peak value, is plotted as a function of $\mathrm{H}_{w}$ which is the field in which the ND patterns are recorded in each warming cycle (figure 13). Expectedly, the martensite phase fraction decreases at higher warming fields.

\subsection{Half-doped charge ordered compound $\mathrm{La}_{0.5} \mathrm{Ca}_{0.5} \mathrm{MnO}_{3}$}

3.2.1. DC Magnetization $\mathrm{La}_{0.5} \mathrm{Ca}_{0.5} \mathrm{MnO}_{3}$ (LCMO) has been widely studied and its properties have been well-documented by several authors. Therefore, we present here only those results which are new and form the basis for very interesting physics. The behavior of magnetization with temperature is similar to that observed in earlier studies $[12,14,21,22,23$. The notable features are that (i) FCC and FCW curves at lower fields show thermal hysteresis accompanying the first order ferromagnetic to antiferromagnetic 
transition, (ii) magnitude of this thermal hysteresis gradually decreases at higher fields, culminating in kinetic arrest of the first order phase transition and the ferromagnetic order persists down to the lowest temperature, (iii) on application of the CHUF protocol (figure 14), devtrification of the arrested, metastable, ferromagnetic phase to the equilibrium antiferromagnetic state and the subsequent reentrance to the stable ferromagnetic state at a higher temperature is observed. Figure 15 shows the magnetization curves measured in different warming fields after cooling from $300 \mathrm{~K}$ in a $6 \mathrm{~T}$ field along with the $6 \mathrm{~T}$ FCC curve. For the $0.01 \mathrm{~T}$ FCW curve, the kinetically arrested ferromagnetic phase devitrifies by about $90 \%$ at $5 \mathrm{~K}$. Subsequently, the remaining FM phase fraction further devitrifies at $\sim 25 \mathrm{~K}$ into the stable low temperature antiferromagnetic phase. On further heating, the antiferromagnetic state reenters the higher temperature ferromagnetic phase at $\sim 230 \mathrm{~K}$ and then, finally goes into the paramagnetic phase. This behavior unfolds more explicitly at higher warming fields. It is also observed that the extent to which the kinetically arrested FM phase devitrifies becomes lesser with higher warming fields and that the temperature at which the devitrified phase reenters the stable ferromagnetic phase decreases. This is clear from the higher moment values at $5 \mathrm{~K}$ for higher warming fields. For warming fields above 3 Tesla, the kinetically arrested phase no longer devitrifies at any intermediate temperature.

3.2.2. Neutron Diffraction Half-doped LCMO has been extensively studied using neutron diffraction [13, 24, 25, 26, 27, 28,. While some of the reports concentrate on determining the magnetic and crystalline aspects of the structure at different temperatures [13], some deal with the local structure and importance of the Mn-O bond lengths [26]. Magnetic field-induced melting of the charge ordered state [27] and phase coexistence of antiferromagnetic and ferromagnetic states due to supercooling [28] have also been discussed. In the present neutron diffraction measurements, we show that the FOPT from high temperature ferromagnetic state to a low temperature antiferromagnetic state is completely or partially arrested (depending on the path taken during cooling in a magnetic field) due to hindered kinetics, resulting in glass-like arrested states or GLAS. We also show that by adopting the CHUF protocol, there is a phase coexistence of GLAS and equilibrium (transformed) states and a de-arrest of the metastable states takes place as one crosses the $\left(\mathrm{H}_{K}, \mathrm{~T}_{K}\right)$ line. Figure 15 shows the Rietveld fitted patterns of LCMO at $295 \mathrm{~K}$ and $9 \mathrm{~K}$. The ND pattern at $295 \mathrm{~K}$ has been refined using the Pnma space group. Refinement of the ND pattern at $9 \mathrm{~K}$ was treated in the manner proposed by Radaelli et al [13] using the space group Pnma. The various parameters deduced from the Rietveld analysis are given in table 1. As expected, magnetic peaks corresponding to CE-type of ordering are observed at $9 \mathrm{~K}$. This antiferromagnetic ordering is characterized by two Mn sublattices [24, $\mathrm{Mn}^{3+}$ and $\mathrm{Mn}^{4+}$. The propagation vectors are $[1 / 2,0,1 / 2]$ for $\mathrm{Mn}^{4+}$ and $[1 / 2,0,0]$ for $\mathrm{Mn}^{3+}$. It is also observed that a small amount of the room temperature phase persists at $9 \mathrm{~K}$ and hence, this was added as a minority phase to the refinement along with its corresponding 
ferromagnetic phase. The refinement shows that the percentage of this minority phase is about $6 \%$. Refinement of the magnetic moments shows that they are in the $a-c$ plane, and $\mu_{x}$ and $\mu_{y}$ were refined separately for $\mathrm{Mn}^{3+}$ while they were constrained to be parallel to $c$-axis for $\mathrm{Mn}^{4+}$. The refined moment values and the percentage phase fractions of the CE-AFM and FM phases are given in table 1. Next, the sample was heated to $300 \mathrm{~K}$ and again cooled down to $9 \mathrm{~K}$ in the presence of a magnetic field of 7 Tesla. The ND pattern at $9 \mathrm{~K}$ cooled in 7 Tesla field shown in figure 16, is marked by a complete absence of any peaks signifying the CE-type AFM order seen earlier and a strong enhancement in the intensities of the nuclear peaks, indicating a well-defined ferromagnetic ordering. This is a clear indication that the formation of the CE-type AFM order has been kinetically arrested due to the field cooling of the sample in a field of 7 Tesla. Refinement of the ND pattern at $9 \mathrm{~K}$ cooled in $7 \mathrm{~T}$ using Pnma space group along with a magnetic phase with ferromagnetic order gives a moment of $4.16 \mu_{B}$ on Mn (table 1). This is consistent with the value obtained from magnetization measurements. After measuring at $9 \mathrm{~K}$ in a cooling field of $7 \mathrm{~T}$, the field was reduced to $0.5 \mathrm{~T}$ (CHUF protocol), the sample was warmed from $9 \mathrm{~K}$ in steps. The data is plotted in a sequence in figure 17. It is seen that up to $30 \mathrm{~K}$, the diffraction spectra remain unchanged and there is no signature of any change in the magnetic order, signifying that the sample remains in a kinetically arrested, metastable ferromagnetic state (magnetic GLAS) up to this temperature. At $35 \mathrm{~K}$, there is an abrupt change in the spectrum, marked by the appearance of magnetic peaks corresponding to the CE-type AFM order (marked as asterisks). These peaks become well-defined at $50 \mathrm{~K}$ and have roughly the same intensity as the ones observed in the zero field cooled case at $9 \mathrm{~K}$. Therefore, $35 \mathrm{~K}$ represents the temperature around which the de-arrest or devitrification of the metastable glassy FM phase occurs and the equilibrium AFM order sets in. The percentage de-arrest would then be a function of the cooling and warming fields. In this case, the refinement of the pattern at $35 \mathrm{~K}$ taken during warming in a field of $0.5 \mathrm{~T}$ indicates that the de-arrest is almost complete since the values of the moments on $\mathrm{Mn}^{3+}$ and $\mathrm{Mn}^{4+}$ are similar to the $9 \mathrm{~K}$, ZFC case (table 1). On warming beyond $150 \mathrm{~K}$, the sample re-enters a weak ferromagnetic state before finally entering the paramagnetic state at room temperature.

In the next measurement cycle, the sample was cooled to $9 \mathrm{~K}$ in a field of 7 Tesla and then, the warming field was set to 3 Tesla (CHUF protocol). As in the earlier measurement cycle, the sample remains in a kinetically arrested metastable ferromagnetic state at $9 \mathrm{~K}$ when the external field is reduced to 3 Tesla. However, upon warming in 3 Tesla (figure 18), the devitrification of the kinetically arrested state takes place only around $75 \mathrm{~K}$, as evidenced by the appearance of weak magnetic peaks attributable to CE-type AFM order. In contrast to the earlier field cycle, the intensities of the CE magnetic peaks are mcuh lower, implying a much higher fraction of the kinetically arrested phase remaining throughout this measurement cycle. A refinement of the pattern at $100 \mathrm{~K}$ gives a moment of $3.83 \mathrm{mu}_{B}$ for the arrested FM phase while moments for the devitrified CE-AFM phase come out as $2.02 \mu_{B}$ for both $\mathrm{Mn}^{3+}$ and $\mathrm{Mn}^{4+}$. The AFM peaks disappear upon warming beyond $150 \mathrm{~K}$ and the sample re-enters 
a fairly strong ferromagnetic phase at $175 \mathrm{~K}$, in consonance with the magnetization measurements (figure 14). Refinement of the pattern at $175 \mathrm{~K}$ of this cycle gives a moment of $3.08 \mu_{B}$ for the re-entrant ferromagnetic phase (table 1).

It is known that the Mn-O bond lengths in LCMO at room temperature and in the CE-AFM phase are quite different due to Jahn-Teller distortion [12. Hence, it can be reasonably expected that the kinetically arrested FM phase would have almost undistorted $\mathrm{MnO}_{6}$ octahedra with all the $\mathrm{Mn}-\mathrm{O}$ distances being approximately similar. Table 1 shows the $\mathrm{Mn}-\mathrm{O}$ bond lengths and $\mathrm{Mn}-\mathrm{O}-\mathrm{Mn}$ bond angles at various temperature and field combinations, and some interesting observations can be made from the values of these quantities. The values shown for $300 \mathrm{~K}$ and $9 \mathrm{~K}$ in zero field show the expected changes in the bond lengths and angles at low temperature. But for the kinetically arrested FM phase at $9 \mathrm{~K}$ obtained by cooling in a field of $7 \mathrm{~T}$ and also when the field is reduced to $0.5 \mathrm{~T}(9 \mathrm{~K}, 0.5 \mathrm{~T})$, the bond angles and lengths do not show any major differences with the $300 \mathrm{~K}$ values. A direct effect of the devitrification of the arrested phase at $35 \mathrm{~K}, 0.5 \mathrm{~T}$ is seen in the Mn-O1 bond length and Mn-O1-Mn bond angle, which are comparable to the values of the $(9 \mathrm{~K}, 0 \mathrm{~T})$ set. A similar behaviour is seen at $100 \mathrm{~K}$ in the $3 \mathrm{~T}$ heating cycle. However, at $175 \mathrm{~K}$ warmed in $3 \mathrm{~T}$ field, there is a re-entrant transition to the equilibrium FM phase, which again shows an undistorted situation with the bond angles and lengths associated with O1 and O2 oxygens being similar.

It is observed that the arrest temperature $\mathrm{T}_{K}$, which has been monitored as the temperature at which the kinetically arrested metastable phase devitrifies, increases with the value of the warming field $\mathrm{H}_{w}$ as was the case with the FSMA sample and this occurs only when the cooling field $\mathrm{H}_{c}$ was greater than the warming field $\mathrm{H}_{w}$. This is consistent with the earlier observation [6] that the condition $\mathrm{H}_{c}>H_{w}$ is required to observe the devitrification whenever the FOPT temperature and the supercooling limit $\mathrm{T}^{*}$ fall with increase in magnetic field. To draw an analogy, these observations are compared with the vitrification of liquid Ge under high pressure [15] wherein it was observed that monoatomic liquid Ge forms a glass when quenched under a pressure of about $10 \mathrm{GPa}$. The metallic Ge glass so obtained transformed, under ambient decompression, from a high density amorphous phase to a low density phase. Similarly, Mishima and Stanley [16] discuss the 1-phase collapse of ice $\mathrm{I}_{h}$ from a high-density liquid to a high-density amorphous phase at high pressures. In the present cases, the transition temperature drops as the magnetic field rises, analogous to the melting point dropping with rising pressure in the case of germanium and water. However, unlike pressure, it is possible to tune a magnetic field effortlessly since it does not require a medium to propagate and use it to study the microscopic nature of magnetic field-induced transitions.

\section{Conclusions}

We have performed neutron diffraction studies on two materials belonging to two different families of compounds, namely the magnetic shape memory alloy 
$\mathrm{Ni}_{37} \mathrm{Co}_{11} \mathrm{Mn}_{42.5} \mathrm{Sn}_{9.5}$ and the charge-ordered manganite $\mathrm{La}_{0.5} \mathrm{Ca}_{0.5} \mathrm{MnO}_{3}$. Using magnetic field as a useful thermodynamic variable and the CHUF protocol, we have shown structural evidence for the first time for (i) the kinetic arrest of the first order phase transition in both compounds, (ii) phase coexistence of the metastable arrested and the equilibrium phases, and (iii) devitrification of the metastable arrested phase.

Our diffraction studies have shown that the arrest temperature rises with increasing field, consistent with an earlier conjecture of Banerjee et al [29] and with an extension of the regime of validity of the Le Chatelier Principle [30]. In cases where the low$\mathrm{T}$ equilibrium phase has a higher magnetization, transition temperature will rise with increasing field, and it was conjectured that the arrest temperature would fall with rising $H$. This has been confirmed through bulk and mesoscopic measurements in some manganites [1, 29, 30], in $\mathrm{Gd}_{5} \mathrm{Ge}_{4}$ [31], and in Ta-doped $\mathrm{HfFe}_{2}$ [32]. Diffraction measurements under the CHUF protocol, with $\mathrm{H}_{w}>H_{c}$ would confirm the earlier

conjecture of Le Chatelier Principle being valid during the devitrification process; some of these materials that are suitable for neutron diffraction studies shall be pursued.

\section{References}

[1] Kranti Kumar, Pramanik A K, Banerjee A, Chaddah P, Roy S B, Park S, Zhang C L, and Cheong S.-W 2006 Phys. Rev. B 73, 184435.

[2] Manekar M A, Chaudhary S, Chattopadhyay M K, Singh K J, Roy S B and Chaddah P 2001 Phys. Rev. B 64, 104416.

[3] Sengupta K and Sampathkumaran E V, 2006 Phys. Rev. B73, 20406.

[4] Banerjee A, Chaddah P, Dash S, Kranti Kumar, Archana Lakhani, Chen X and Ramanujan R V 2011 Phys. Rev. B84, 214420.

[5] Umetsu R Y, Ito K, Ito W, Koyama K, Kanomata T, Ishida K and Kainuma R 2011 J. Alloys and Compd. 509, 1389.

[6] Archana Lakhani, Banerjee A, Chaddah P, Chen X and Ramanujan R V 2012 J. Phys. Condens. Matter 24, 386004 and references therein.

[7] Chaddah P and Banerjee A 2010 arXiv:1004.3116v3 and references therein.

[8] Xu X, Ito W, Tokunaga M, Umetsu R Y, Kainuma R, Ishida K 2010 Mater. Trans. 51, 1357.

[9] Sanchez Llamazares J, Hernando B, Sunol J J, Garcia C and Ross C A 2010 J. Appl. Phys. 107, 09A956.

[10] Sharma V K, Chattopadhyay M K and Roy S B 2007 Phys. Rev. B 76, 140401(R).

[11] Chaddah P and Banerjee A 2012 arXiv:1201.0575 and references therein.

[12] Chaddah P, Kranti Kumar and Banerjee A 2008 Phys. Rev. B 77, 100402(R).

[13] Radaelli P G, Cox D E, Marezio and Cheong S -W 1997 Phys. Rev. B 55, 3015.

[14] Banerjee A, Kranti Kumar and Chaddah P 2008 J. Phys. Condens. Matter 20, 255245; Banerjee A, Kranti Kumar and Chaddah P 2009 J. Phys. Condens. Matter 21, 26002.

[15] Bhat M H, Molinero V, Soignard E, Solomon V C, Sastry S, Yarger J L, Angell C A 2007 Nature 448, 787.

[16] Mishima O and Stanley H E 1998 Nature 396, 329.

[17] Pimpale A V, Dasannacharya B A, Siruguri V, Babu P D and Goyal P S 2002 Nucl. Instrum. Methods A 481, 615.

[18] Watchel E, Henninger F and Predel B 1983 J. Magn. Magn. Mater. 38, 305.

[19] Yuhasz W M, Schlagel D L, Xing Q, McCallum R W and Lograsso T A 2010 J. Alloys Compd. 492, 681 . 
[20] Banerjee A, Dash S, Archana Lakhani, Chaddah P, Chen X and Ramanujan R V, 2011 Solid State Commun. 151, 971.

[21] Loudon J C, Mathur N D and Midgley P A 2002 Nature 420, 797.

[22] Babu P D, Das A, Paranjpe S K 2001 Solid State Commun. 118, 91.

[23] Das A, Babu P D, Chatterjee S and Nigam A K, 2004 Phys. Rev. B 70, 224404.

[24] Wollan E O and Koehler W C, 1955 Phys. Rev. 100, 545.

[25] Goodenough J B, 1955 Phys. Rev. 100, 564.

[26] Rodriguez E E, Proffen Th, Llobet A, Rhyne J J and Mitchell J F 2005 Phys. Rev. B 71, 104430.

[27] Tyson T A, Deleon M, Croft M, Harris V G, Kao C -C, Kirkland J and Cheong S -W 2004 Phys. Rev. B. 70, 24410.

[28] Kallias G, Pissas M and Hoser A 2000 Physica B 276-278, 778.

[29] Banerjee A, Pramanik A K, Kranti Kumar and Chaddah P 2006 J. Phys. Condens. Matter 18, L605; Banerjee A, Mukherjee K, Kranti Kumar and Chaddah P 2006 Phys. Rev. B 74, 224445.

[30] Archana Lakhani, Kushwaha P, Rawat R, Kranti Kumar, Banerjee A and Chaddah P $2010 \mathrm{~J}$. Phys. Condens. Matter 22, 032101.

[31] Roy S B, Chattopadhyay M K, Banerjee A, Chaddah P, Moore J D, Perkins G K, Cohen L F, Gschneider Jr K A and Pecharsky V K, 2007 Phys. Rev. B 75, 184410.

[32] Rawat R, Chaddah P, Bag P, Babu P D and Siruguri V 2013 J. Phys.: Condens. Matter 25, 066011. 
Table 1. Structural parameters of $\mathrm{La}_{0.5} \mathrm{Ca}_{0.5} \mathrm{MnO}_{3}$ obtained from Rietveld refinement of neutron diffraction data taken at different temperatures and magnetic fields. Note that $0.5 \mathrm{~T}$ and $3 \mathrm{~T}$ data are taken in warming cycle after the sample is cooled in 7 $\mathrm{T}$ field from $300 \mathrm{~K}$ to $9 \mathrm{~K}$ and reducing the field isothermally to the respective field values at $9 \mathrm{~K}$.

\begin{tabular}{|c|c|c|c|c|c|c|c|c|}
\hline \multicolumn{2}{|c|}{ Parameter } & $300 \mathrm{~K}, 0 \mathrm{~T}$ & $9 \mathrm{~K}, \mathrm{0T}$ & $9 \mathrm{~K}, 7 \mathrm{~T}$ & $9 \mathrm{~K}, 0.5 \mathrm{~T}$ & $35 \mathrm{~K}, 0.5 \mathrm{~T}$ & $100 \mathrm{~K}, 3 \mathrm{~T}$ & $175 \mathrm{~K}, 3 \mathrm{~T}$ \\
\hline \multicolumn{2}{|c|}{$a$} & $5.4197(4)$ & $5.4409(4)$ & $5.4155(4)$ & $5.4142(4)$ & $5.4398(4)$ & $5.4409(4)$ & $5.4169(4)$ \\
\hline \multicolumn{2}{|c|}{$b$} & $7.6462(5)$ & $7.5280(5)$ & $7.6363(5)$ & $7.6354(5)$ & $7.5305(5)$ & $7.5303(5)$ & $7.6334(5)$ \\
\hline \multicolumn{2}{|c|}{$c$} & $5.4268(4)$ & $5.4741(4)$ & $5.4210(4)$ & $5.4202(4)$ & $5.4711(4)$ & $5.4767(4)$ & $5.4247(4)$ \\
\hline \multicolumn{2}{|c|}{$\mathrm{V}$} & $224.89(5)$ & $224.21(6)$ & $224.18(5)$ & $224.08(5)$ & $224.12(8)$ & $224.39(9)$ & $224.31(5)$ \\
\hline \multicolumn{2}{|c|}{$\mathrm{La} / \mathrm{Ca}$ x } & $0.0147(4)$ & $0.0228(4)$ & $0.0211(3)$ & $0.0215(5)$ & $0.0153(4)$ & $0.0162(3)$ & $0.0239(4)$ \\
\hline & $\mathrm{z}$ & $0.4958(3)$ & $0.4958(3)$ & $0.4964(1)$ & $0.4961(5)$ & $0.4988(1)$ & $0.4950(3)$ & $0.4982(5)$ \\
\hline \multirow[t]{2}{*}{ O1 } & $\mathrm{x}$ & $0.4931(3)$ & $0.4941(4)$ & $0.4893(3)$ & $0.4928(3)$ & $0.4907(4)$ & $0.5000(5)$ & $0.4922(3)$ \\
\hline & $\mathrm{z}$ & $0.5617(3)$ & $0.5660(4)$ & $0.5584(3)$ & $0.5584(3)$ & $0.5629(4)$ & $0.5705(4)$ & $0.5574(3)$ \\
\hline \multirow[t]{3}{*}{$\mathrm{O} 2$} & $\mathrm{x}$ & $0.2759(3)$ & $0.2696(4)$ & $0.2774(3)$ & $0.2751(4)$ & $0.2689(4)$ & $0.2764(4)$ & $0.2758(4)$ \\
\hline & $\mathrm{y}$ & $0.0319(5)$ & $0.0334(6)$ & $0.0338(5)$ & $0.0337(6)$ & $0.0332(6)$ & $0.0350(5)$ & $0.0339(5)$ \\
\hline & z & $0.2224(3)$ & $0.2275(4)$ & $0.2240(3)$ & $0.2205(4)$ & $0.2261(4)$ & $0.2212(4)$ & $0.2233(4)$ \\
\hline \multicolumn{2}{|c|}{ B } & $0.64(7)$ & $0.18(8)$ & $0.03(8)$ & $0.05(8)$ & $0.58(9)$ & $0.14(8)$ & $0.14(8)$ \\
\hline \multicolumn{2}{|c|}{ Mn-O1 } & $1.9410(4)$ & $1.9167(6)$ & $1.9360(4)$ & $1.9353(5)$ & $1.9145(8)$ & $1.9218(9)$ & $1.9340(4)$ \\
\hline \multirow{2}{*}{\multicolumn{2}{|c|}{$\mathrm{Mn}-\mathrm{O} 2$}} & $1.9371(4)$ & $1.9403(6)$ & $1.9388(4)$ & $1.9270(5)$ & $1.9316(7)$ & $1.9490(8)$ & $1.9404(4)$ \\
\hline & & $1.9505(4)$ & $1.9649(6)$ & $1.9487(4)$ & $1.9605(6)$ & $1.9722(8)$ & $1.9700(9)$ & $1.9483(5)$ \\
\hline \multicolumn{2}{|c|}{ Mn-O1-Mn } & $160.02(8)$ & $158.17(9)$ & $160.85(8)$ & $161.05(8)$ & $159.07(10)$ & $156.81(10)$ & $161.31(8)$ \\
\hline \multicolumn{2}{|c|}{$\mathrm{Mn}-\mathrm{O} 2-\mathrm{Mn}$} & $161.09(6)$ & $162.37(7)$ & $160.50(6)$ & $160.36(6)$ & $162.34(8)$ & $160.08(8)$ & $160.56(6)$ \\
\hline \multicolumn{3}{|c|}{ Magnetic order } & $\mathrm{AFM}$ & FM & $\mathrm{FM}$ & $\mathrm{AFM}+\mathrm{FM}$ & $\mathrm{AFM}+\mathrm{FM}$ & FM \\
\hline \multicolumn{3}{|c|}{$\mu_{x}\left(\mu_{B}\right)$} & $1.00(6)$ & & & $1.00(6)$ & & \\
\hline \multicolumn{2}{|c|}{$\mu_{z}\left(\mu_{B}\right)$} & \multicolumn{7}{|c|}{$\mathrm{Mn}^{4+}:$} \\
\hline \multicolumn{2}{|c|}{$\mu_{z}\left(\mu_{B}\right)$} & & $2.46(5)$ & & & $2.26(5)$ & $2.20(10)$ & \\
\hline \multicolumn{2}{|c|}{$\mu_{z}\left(\mu_{B}\right)$} & & $3.25(8)$ & $4.17(4)$ & $3.96(4)$ & $3.73(4)$ & $3.83(6)$ & $3.08(7)$ \\
\hline \multicolumn{2}{|c|}{$\mathrm{R}_{w p}$} & 10.7 & 19.0 & 16.1 & 19.7 & 16.5 & 25.7 & 23.4 \\
\hline \multicolumn{2}{|c|}{$\mathrm{R}_{\text {Bragg }}$} & 8.9 & 10.4 & 6.8 & 7.7 & 7.6 & 11.1 & 8.1 \\
\hline
\end{tabular}




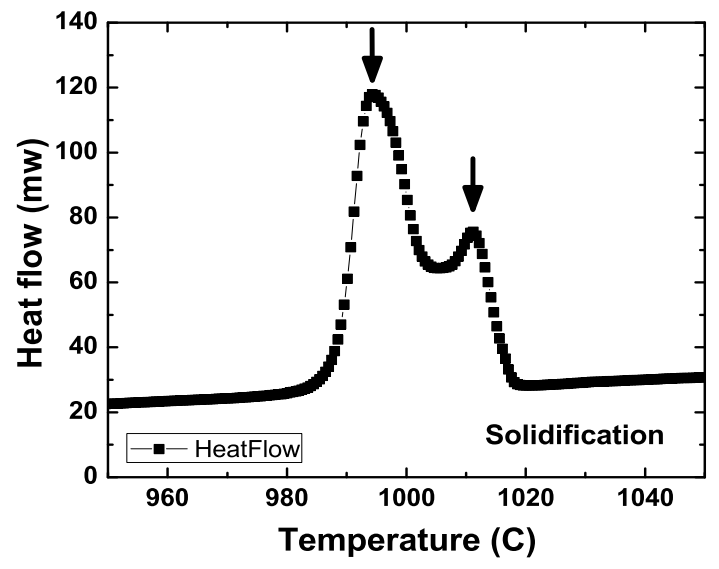

Figure 1. High temperature DSC plots of $\mathrm{Ni}_{37} \mathrm{Co}_{11} \mathrm{Mn}_{42.5} \mathrm{Sn}_{9.5}$ showing the two-phase incongruent melting behavior.

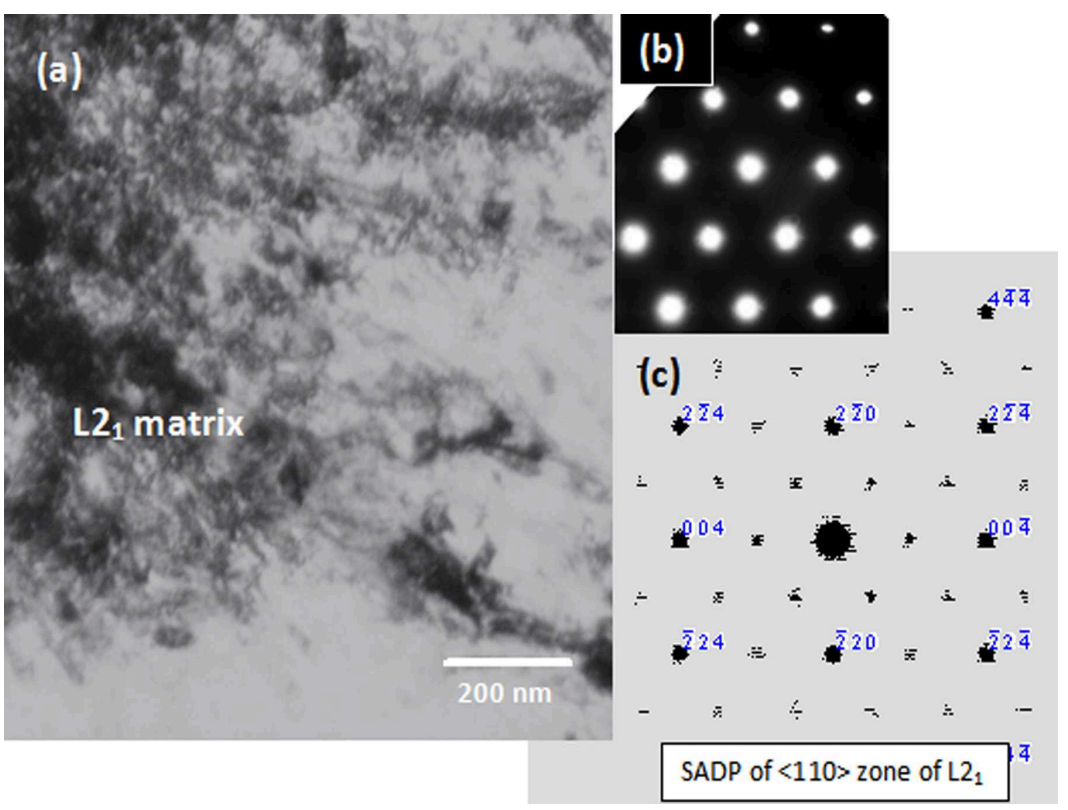

Figure 2. (a) Microstructure of the parent $\mathrm{L} 2{ }_{1}$ phase in as-solutionized condition, (b) SAD pattern of the $\langle 110\rangle$ zone axis, and (c) its simulated key. 


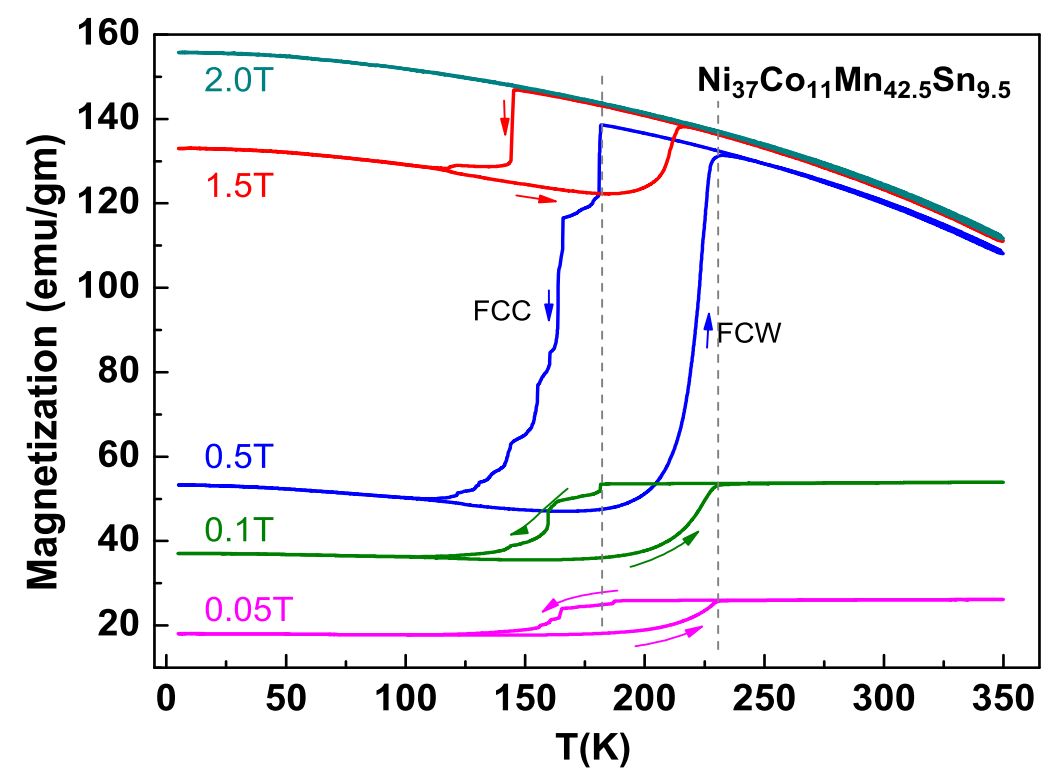

Figure 3. $\mathrm{M}$ versus $\mathrm{T}$ plots showing the field-cooled cooling (FCC) and field-cooled warming (FCW) curves in applied fields of 0.05, 0.1, 0.5, 1.5 and 2 Tesla.

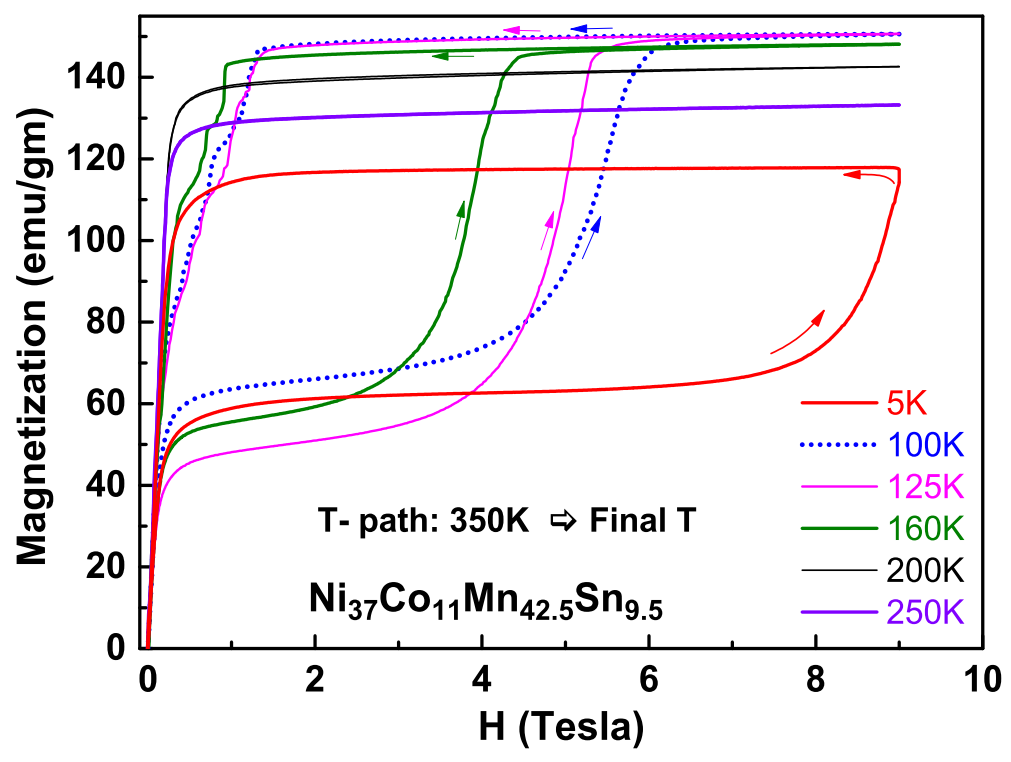

Figure 4. $\mathrm{M}$ versus $\mathrm{H}$ isotherms at various temperatures after cooling the sample from $350 \mathrm{~K}$. 


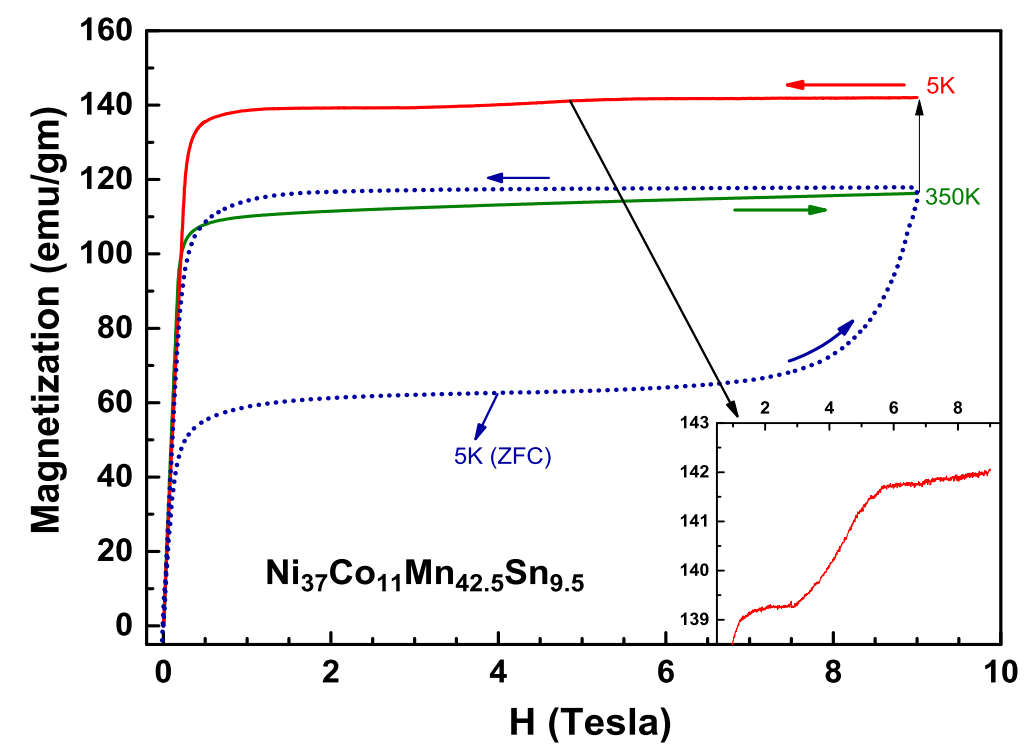

Figure 5. Field-cooled $\mathrm{MH}$ isotherms where field is raised to $9 \mathrm{~T}$ at $350 \mathrm{~K}$ (green curve) and sample cooled to $5 \mathrm{~K}$ in field after which the field is reduced to zero (red curve) during measurement. ZFC-MH (blue curve) at $5 \mathrm{~K}$ is also shown for comparison. Inset shows the magnified part of $\mathrm{FC}-\mathrm{MH}$ at $5 \mathrm{~K}$, which shows the devitrification of the arrested high temperature phase.

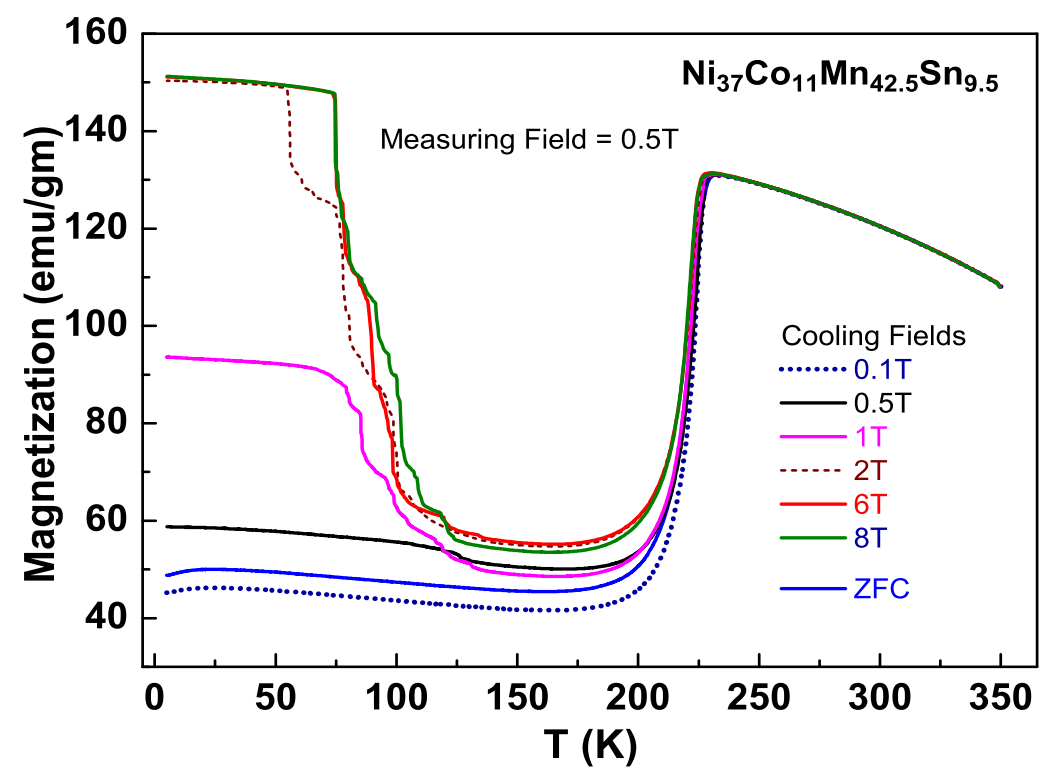

Figure 6. Magnetization as a function of temperature using the CHUF protocol. The sample is cooled in various fields and measured in $0.5 \mathrm{~T}$ in the warming cycle. 


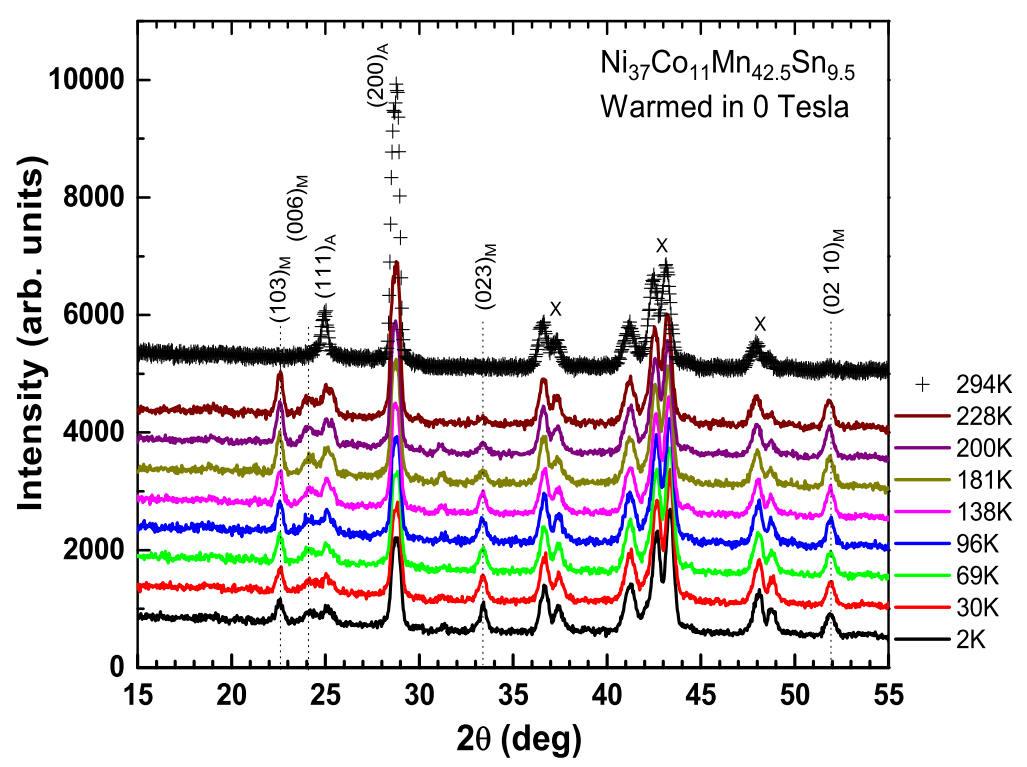

Figure 7. Neutron diffraction patterns as a function of temperature. Indices with subscript 'A' belong to the austenite phase while those with subscript ' $M$ ' belong to the $10 \mathrm{M}$ martensite phase. Peaks in the regions marked as ' $\mathrm{X}$ ' are contributions from the magnet shroud. Patterns have been offset for clarity.

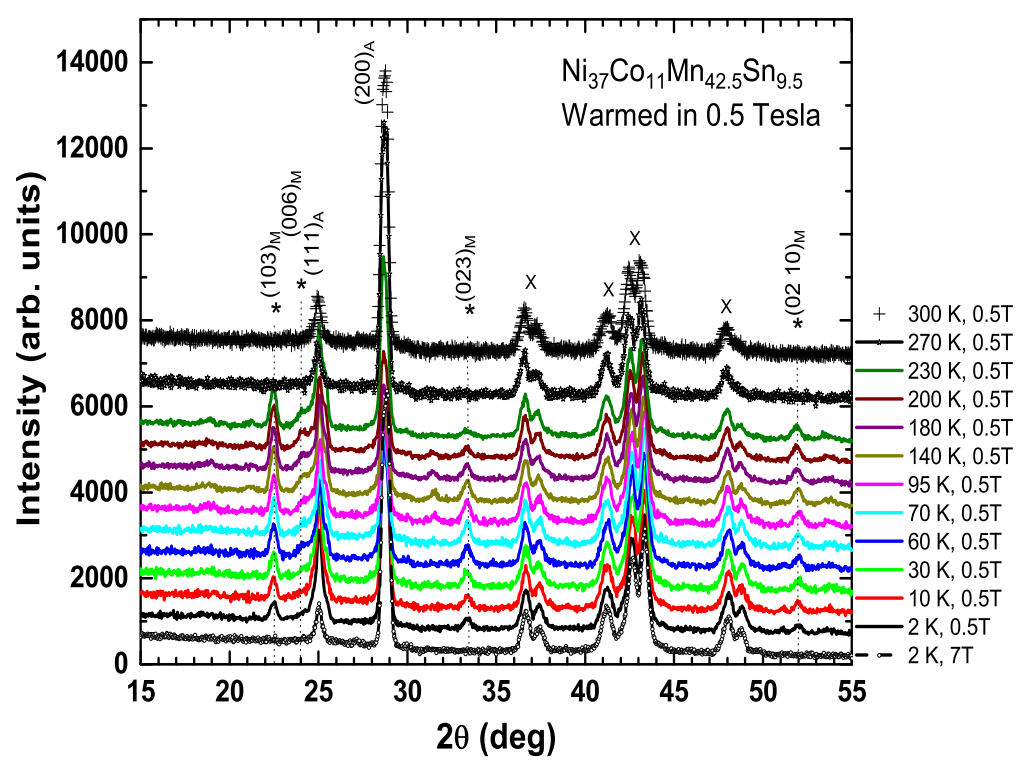

Figure 8. Neutron diffraction patterns obtained using the CHUF protocol. The sample was cooled from $300 \mathrm{~K}$ to $2 \mathrm{~K}$ in a field of 7 Tesla which is isothermally reduced to 0.5 T. Data were taken in the warming cycle. Subscripts A, M and X have the same meaning as in Fig. 7 . 


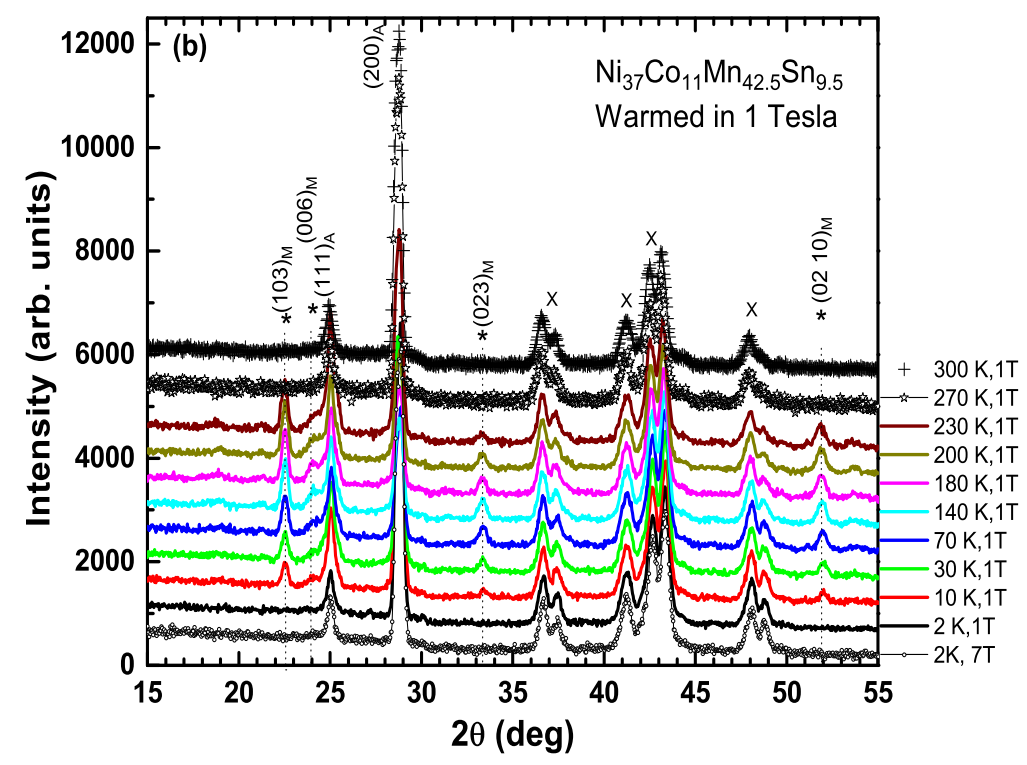

Figure 9. Neutron diffraction patterns obtained using the CHUF protocol. The sample was cooled from $300 \mathrm{~K}$ to $2 \mathrm{~K}$ in a field of 7 Tesla which is isothermally reduced to $1 \mathrm{~T}$. Data were taken in the warming cycle. Subscripts A, M and X have the same meaning as in Fig. 7.

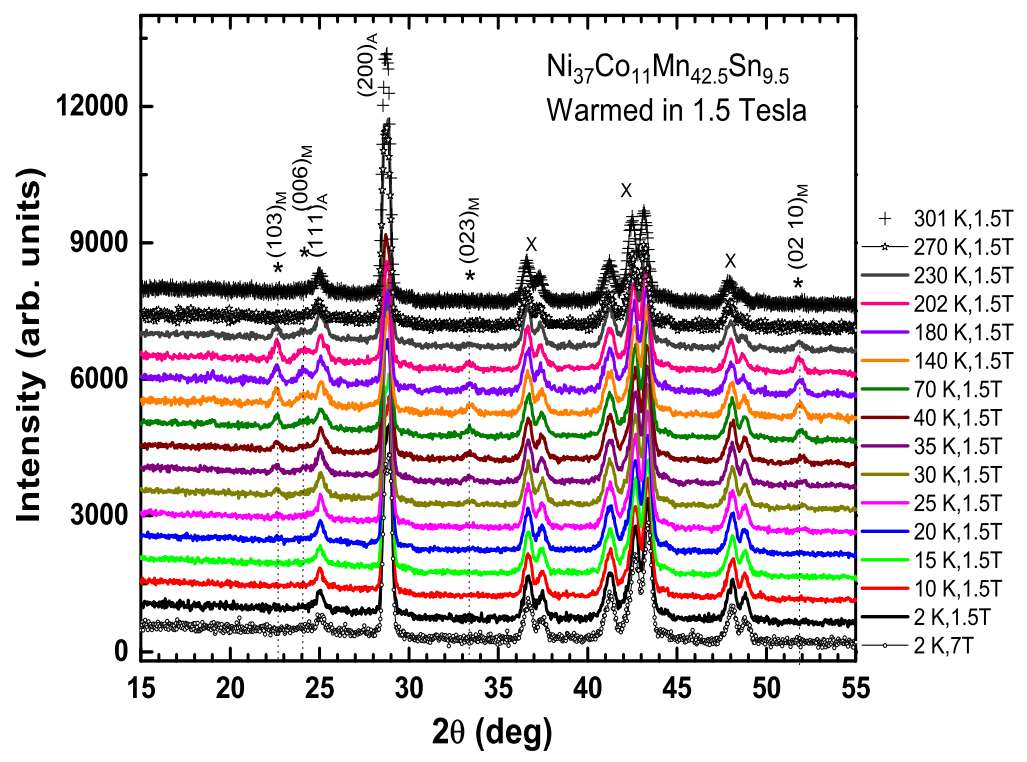

Figure 10. Neutron diffraction patterns obtained using the CHUF protocol. The sample was cooled from $300 \mathrm{~K}$ to $2 \mathrm{~K}$ in a field of 7 Tesla which is isothermally reduced to $1.5 \mathrm{~T}$. Data were taken in the warming cycle. Subscripts A, M and X have the same meaning as in Fig. 7. 


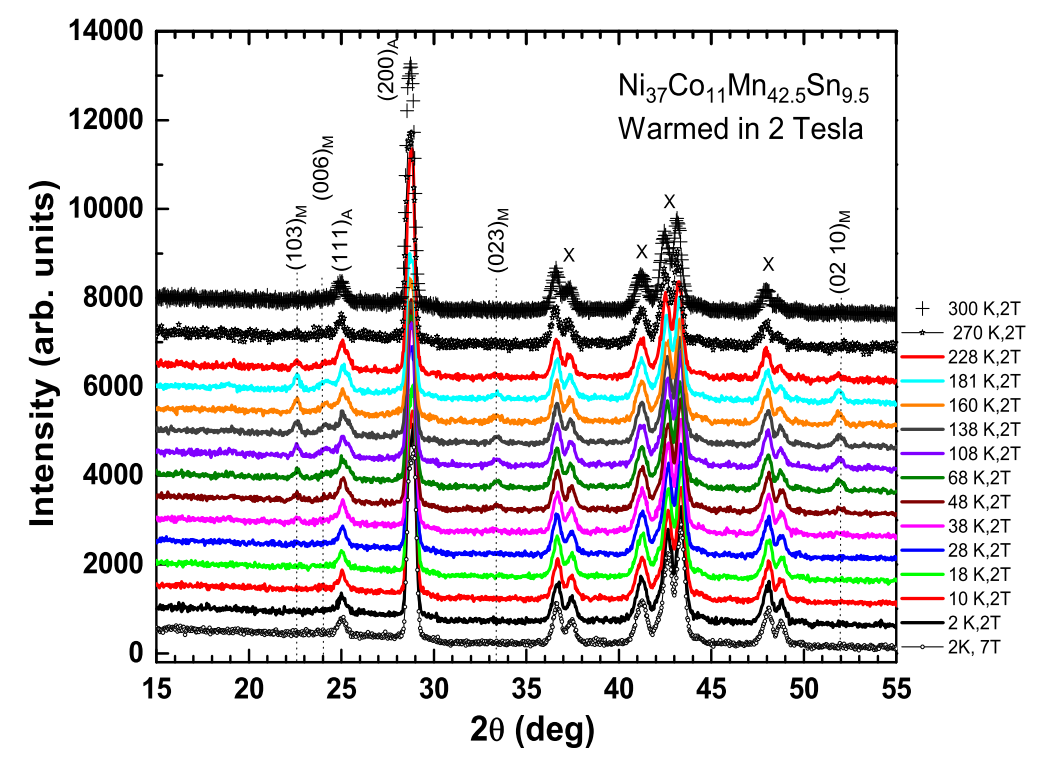

Figure 11. Neutron diffraction patterns obtained using the CHUF protocol. The sample was cooled from $300 \mathrm{~K}$ to $2 \mathrm{~K}$ in a field of 7 Tesla which is isothermally reduced to $2 \mathrm{~T}$. Data were taken in the warming cycle. Subscripts A, M and X have the same meaning as in Fig. 7.

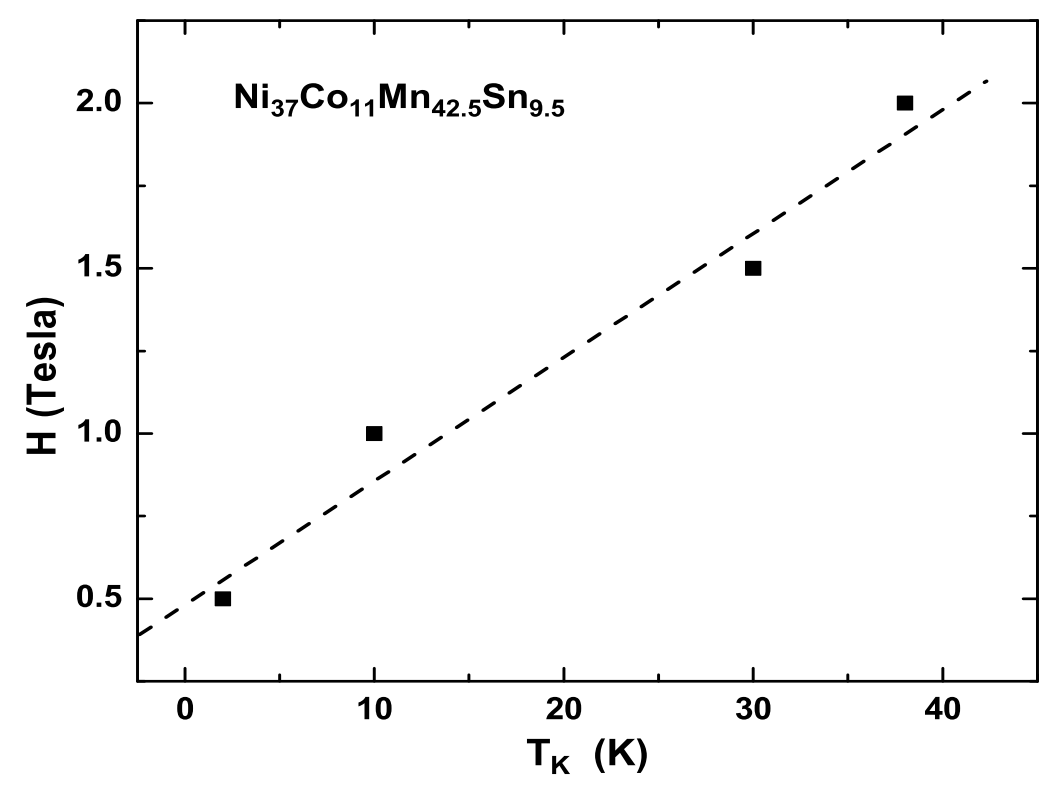

Figure 12. H-T diagram showing the kinetic arrest line across which there would be a devitrification from the arrested metastable austenite phase to the equilibrium martensite phase. 


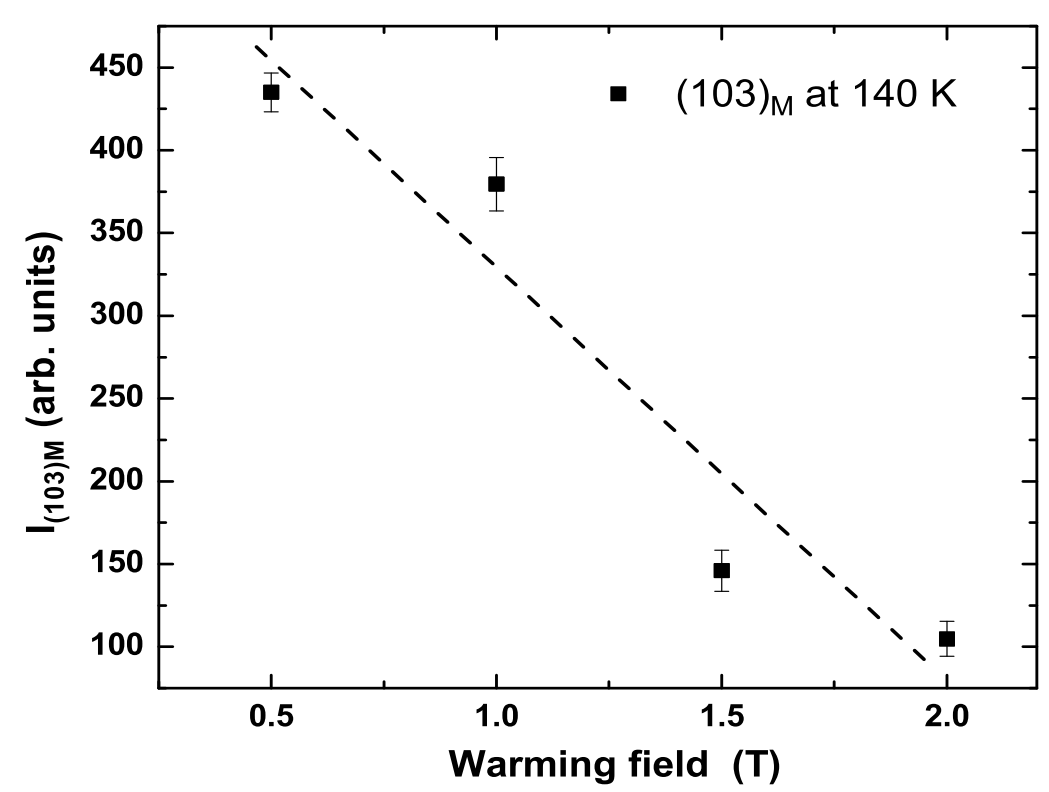

Figure 13. Integrated intensity of the (103) reflection of the $10 \mathrm{M}$ martensite phase (obtained from figures 9-11) as a function of the warming field at $140 \mathrm{~K}$.

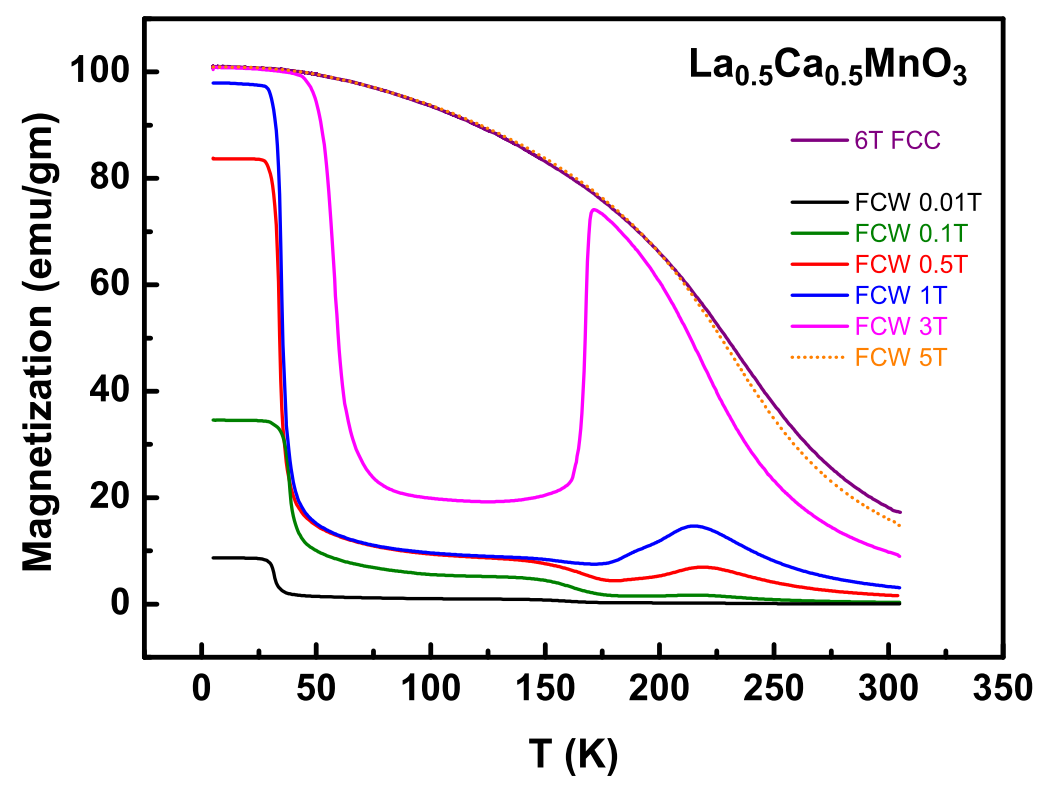

Figure 14. Magnetization as a function of temperature employing the CHUF protocol when the sample LCMO is cooled in a field of $6 \mathrm{~T}$ and measurements are carried out in different warming fields. 


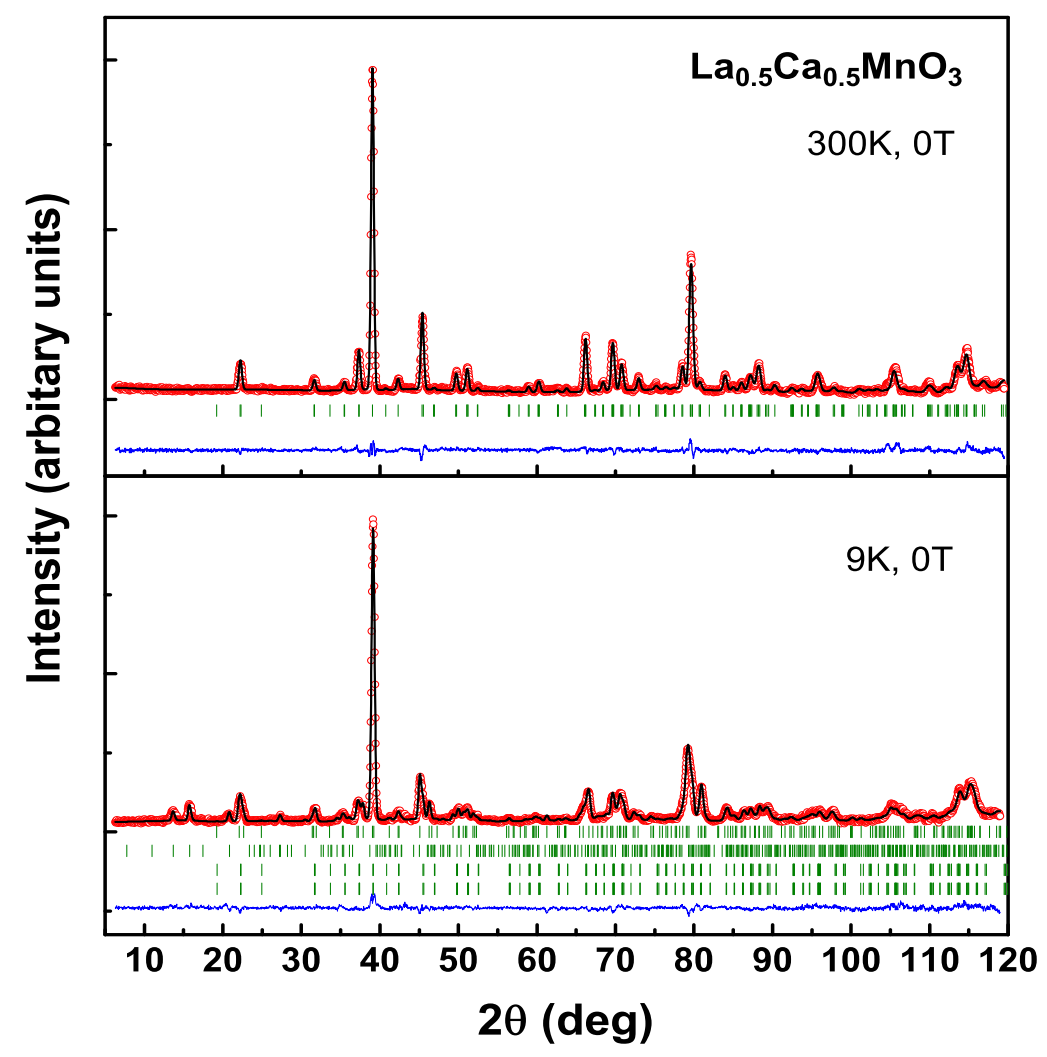

Figure 15. Rietveld fitted plots of LCMO at $300 \mathrm{~K}$ and $9 \mathrm{~K}$ measured in zero field. Observed data are filled circles and the calculated pattern is shown as continuous line through the data points. Difference pattern along with the Bragg ticks are shown below the patterns. For $9 \mathrm{~K}$ data, Bragg ticks indicate the phases (from top) majority nuclear phase, CE-AFM phase, nuclear and FM phases of the high temperature residual phase.

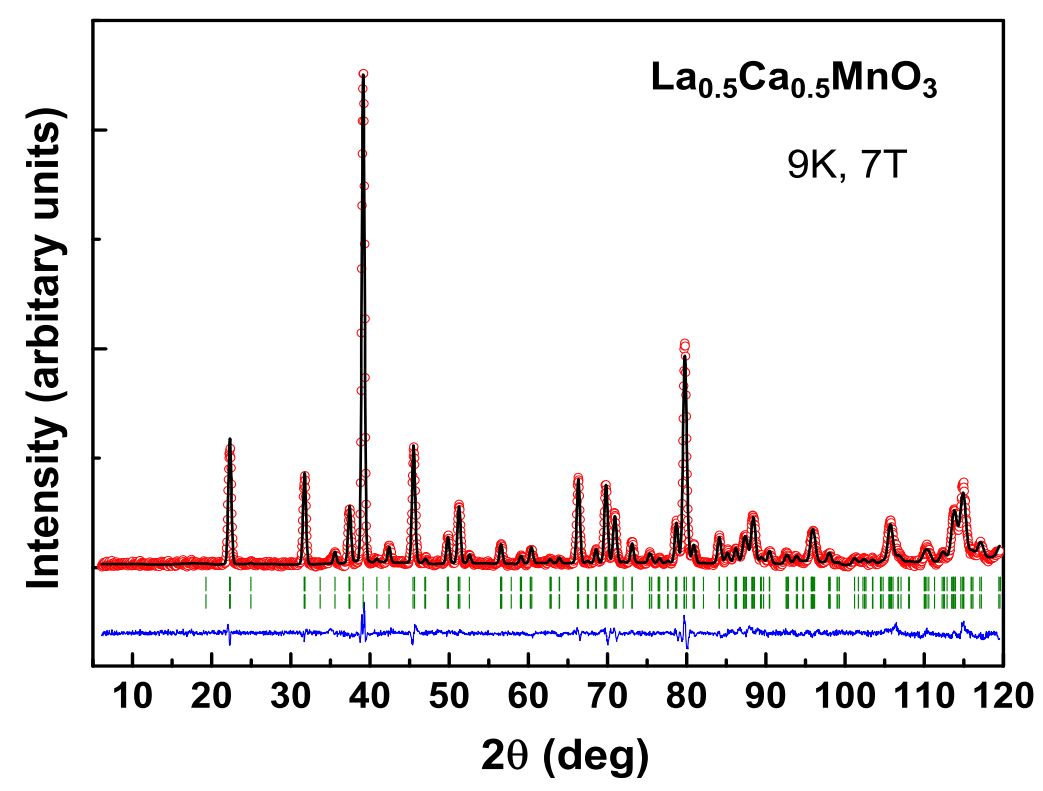

Figure 16. Rietveld refined pattern of the kinetically arrested metastable FM phase at $9 \mathrm{~K}$, cooled in a field of $7 \mathrm{~T}$. Bragg ticks indicate the nuclear and FM phases. 


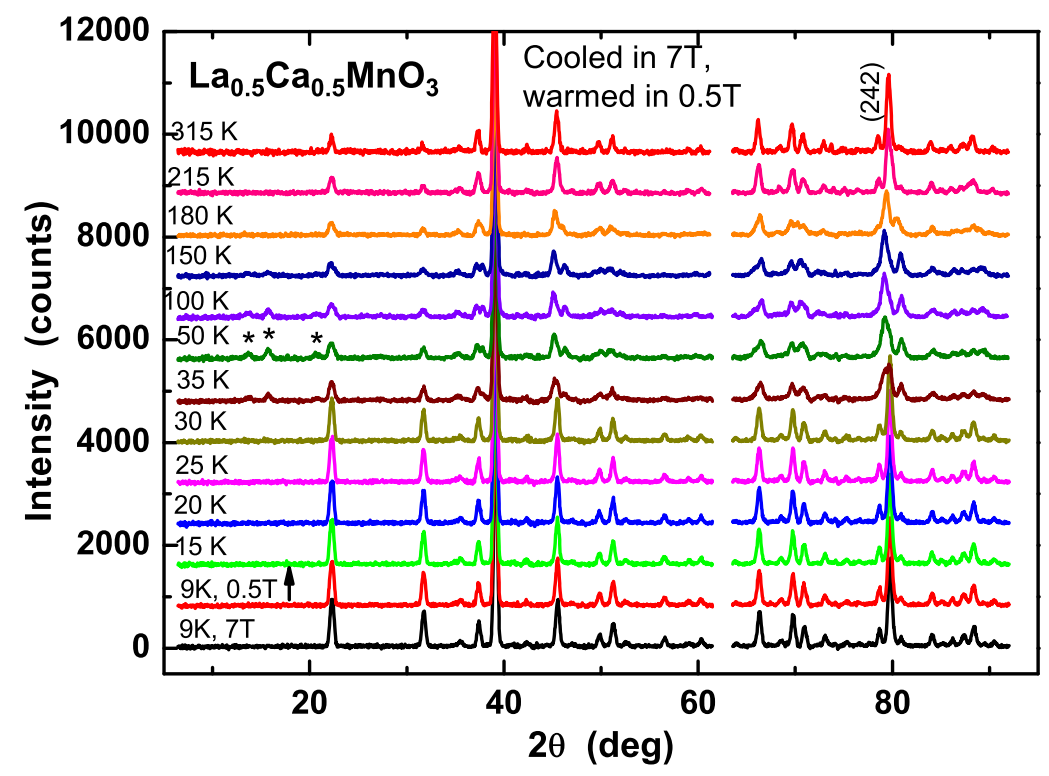

Figure 17. Neutron diffraction patterns obtained using the CHUF protocol. The sample was cooled from $300 \mathrm{~K}$ to $9 \mathrm{~K}$ in a field of 7 Tesla which is isothermally reduced to $0.5 \mathrm{~T}$. Data were taken in the warming cycle. Pattern taken at $9 \mathrm{~K}, 7 \mathrm{~T}$ is shown at the bottom as a reference. Asterisks indicate the positions of the CE-AFM peaks observed upon the devitrification of the arrested FM phase.

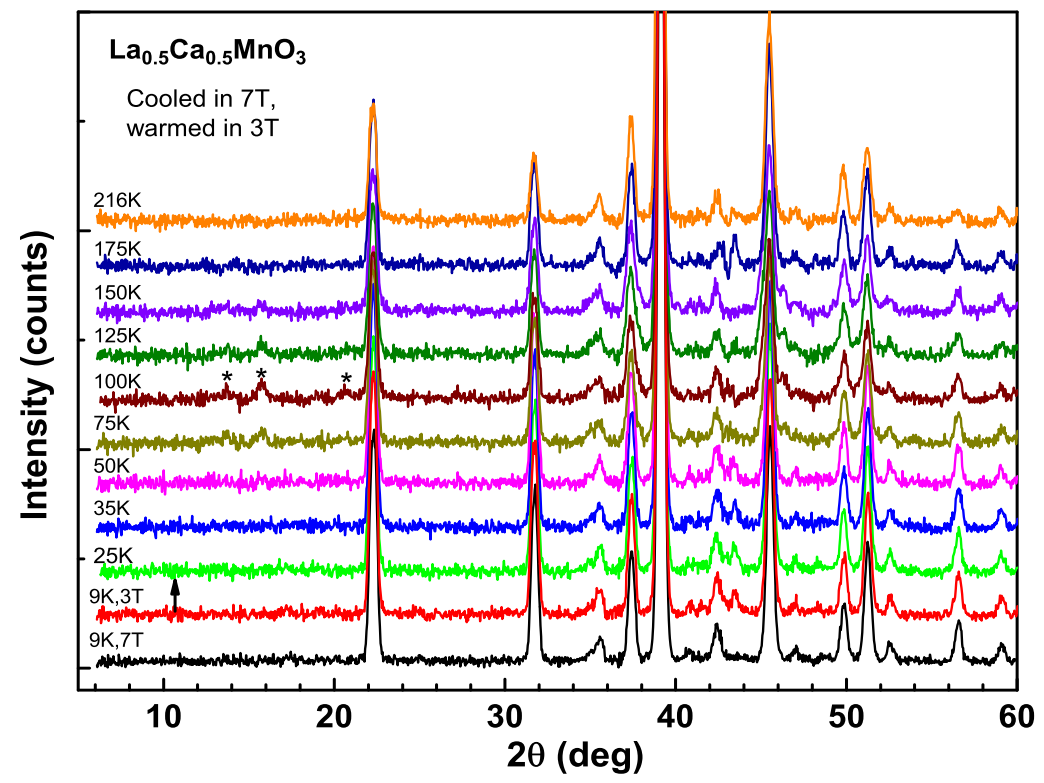

Figure 18. Neutron diffraction patterns obtained using the CHUF protocol. The sample was cooled from $300 \mathrm{~K}$ to $9 \mathrm{~K}$ in a field of 7 Tesla which is isothermally reduced to $3 \mathrm{~T}$. Data were taken in the warming cycle. Pattern taken at $9 \mathrm{~K}, 7 \mathrm{~T}$ is shown at the bottom as a reference. Asterisks indicate the positions of the CE-AFM peaks observed upon the devitrification of the arrested FM phase. 